\title{
DEPLETION FORCES IN COLLOIDAL SUSPENSIONS
}

\author{
D. Henderson ${ }^{1, *}$, S. Sokolowski ${ }^{2}$, D. Wasan ${ }^{3}$ \\ ${ }^{1}$ Department of Chemistry and Biochemistry, Brigham Young University, Provo UT 84602, USA \\ ${ }^{2}$ Department for the Modelling of Physico-Chemical Properties, \\ Marie Curie-Sklodowska University, 20031 Lublin, Poland \\ ${ }^{3}$ Illinois Institute of Technology, Chicago IL 60616, USA
}

(Received March 31, 1998)

\begin{abstract}
The classical theory for the interactions between colloidal particles is the DLVO theory in which the interaction is presumed to consist of a repulsive long range electrostatic term and an attractive short range dispersion term. One important missing term is the effect of the depletion force (known in the statistical mechanics community as the volume exclusion force). An approximate description of this term was obtained by Henderson and Lozada Cassou (HCL) from the theory of hard sphere mixtures. There has been some controversy about the manner in which this term was obtained. Some aspects of this question are discussed and an account of some recent work that has settled this controversy and that justifies the original result of HLC are reviewed.
\end{abstract}

Key words: colloids, mixtures, forces of interaction, integral equations.

PACS number(s): 61.20.Gy, 61.20.Ne, 82.70.Dd

\section{INTRODUCTION}

The classical theory of the interaction between colloidal particles is that of Derjaguin, Landau, Verwey, and Overbeek (DLVO) in which the interaction energy between two colloidal particles, of radius, $a$, is given by two terms. One is a long range repulsive electrostatic interaction and the other is a short range attractive dispersion (or van der Waals or Hamaker) interaction. Thus

$$
\frac{W_{c c}(r)}{a}=\frac{A}{H}+B \exp (-\kappa H)
$$

where $A$ and $B$ are constants that can be obtained from experiment,

$$
\kappa^{2}=\frac{4 \pi \beta e^{2}}{\varepsilon} \sum_{i} z_{i}^{2} \rho_{i}
$$

$\beta=1 / k T$ ( $k$ is Boltzmann's constant and $T$ is the temperature), $e$ is the electronic charge, $\varepsilon$ is the dielectric constant of the supporting fluid, and $z_{i}$ and $\rho_{i}=N_{i} / V$ ( $N_{i}$ is the number of particles of species $i$ and $V$ is the volume) are, respectively, the valence and density of the charged particles. Thus, $\kappa$ is the usual Debye screening parameter. For simplicity, we assume that the concentration of the colloidal particles is vanishingly small (added salt), and the sum in eq. (2) is over the ions in the supporting fluid. Finally, $H$ is the separation of the surfaces of the colloidal particles; thus, $H=r-2 a$, where $r$ is the distance between the centers of the colloidal particles. For simplicity, we have assumed that all the colloidal particles have the same diameter. The extension to the case of colloidal particles of varying size is complex but straightforward in principle.

It is important to note that in the DLVO theory, the interaction energy diverges as $a$ increases but that the interaction energy divided by $a$ is finite. Equation (1) gives a pleasing description of the stability or flocculation of colloidal suspensions. At long range, the interaction is repulsive; at shorter range there is a maximum and then the interaction is repulsive. If the peak is high enough, the suspension is stable. However, if the peak is relatively small, flocculation occurs.

This is, indeed, a satisfying picture. Unfortunately, nature intrudes. The experiments of Israelachvili [1] and those of own laboratory [2] show that the DLVO theory is incomplete. At short separations, the interaction is oscillatory. This oscillatory force can be described by the formalism used in the statistical mechanics of liquids.

\section{ORNSTEIN-ZERNIKE EQUATION}

The description of the depletion force is well known in the theory of liquids, where it is known as the volume exclusion force. The idea of volume exclusion dates back to van der Waals who introduced the 'free volume', $V-b$, in his famous equation of state. The 'free volume' differs from the actual volume, $V$, because some of the volume is excluded by the volume occupied by the molecules, which is more or less $b$ except that a factor of 2 must be introduced to account for the fact that the excluded volume of a pair of molecules is shared.

The van der Waals expression is too drastic. It is correct only in one dimension. In two or more dimensions, 
a molecule can pass over another molecule, resulting in a smaller 'free volume' that is equal to $V-b$ only at extremely low densities. The 'free volume' is a complex function of the density.

The modern theory of liquids (and dense gases) is based upon the Ornstein-Zernike (OZ) equation

$$
h_{i j}\left(r_{12}\right)-c_{i j}\left(r_{12}\right)=\sum_{k} \rho_{k} \int d \mathbf{r}_{3} h_{i k}\left(r_{13}\right) c_{k j}\left(r_{32}\right)
$$

where $r_{i j}=\left|\mathbf{r}_{i}-\mathbf{r}_{j}\right|$ is the separation of a pair of particles whose centers are at $\mathbf{r}_{i}$ and $\mathbf{r}_{j}$. The function $h_{i j}(r)$ is the total correlation function (TCF) of a pair of particles of species $i$ and $j$ and is given by

$$
h_{i j}(r)=g_{i j}(r)-1
$$

where $g_{i j}(r)$ is the radial distribution function (RDF) of a pair of particles of species $i$ and $j$. The RDF gives the probability of finding such a pair at a distance $r$, normalized so that

$$
\lim _{r \rightarrow \infty} g_{i j}(r) \rightarrow 1
$$

In the OZ equation, the TCF is divided in two terms. The first term is the direct correlation function (DCF) that describes the correlations between two particles directly. The second term is the indirect term that describes the correlations between two particles through the presence of a third one.

Equation (3) is nothing more than a definition of the DCF. If either the TCF or DCF is known, the other may be obtained. However, if they are not known then the $\mathrm{OZ}$ equation does not provide a method of obtaining them unless something new is added.

The new element is a closure that gives an additional relation between the TCF and the DCF. The most important simple closure is the hypernetted chain (HNC) approximation [3],

$$
\gamma_{i j}(r)=\ln y_{i j}(r)
$$

where

$$
\gamma_{i j}(r)=h_{i j}(r)-c_{i j}(r)
$$

is the sum of integrals in the OZ equation. The other function is called the cavity or background function and is

$$
y_{i j}(r)=\exp \left[\beta u_{i j}(r)\right] g_{i j}(r)
$$

where $u_{i j}$ is the pair interaction potential between a pair of molecules of species $i$ and $j$. Equation (6) is valid at low densities. The HNC approximation consists of presuming that this result is applicable at higher densities.
Care must be taken in defining $y_{i j}(r)$ in regions where $u_{i j}(r)$ is large and positive. However, this can be done.

Equation (6) can be linearized to yield the PercusYevick (PY) approximation [4],

$$
\gamma_{i j}(r)=y_{i j}(r)-1
$$

Another manner of linearizing eq. (6) yields the mean spherical approximation (MSA [5],

$$
\begin{gathered}
g_{i j}(r)=-1, r<\sigma_{i j}, \\
g_{i j}(r)=-\beta u_{i j}(r), r>\sigma_{i j},
\end{gathered}
$$

where $\sigma_{i j}$ is the hard core distance of the interaction between a pair of particles of species $i$ and $j$.

The PY approximation is useful for hard spheres and hard sphere mixtures but not much else. However, for hard spheres it is quite accurate and has the virtue of yielding analytical results. The HNC approximation is not very useful for hard spheres (except in one instance that we will describe in this note) but is useful for fluids with a longer range potential, such as charged fluids and fluids with dipole moments, as for example, water.

The MSA seems to be a sort of cross between the PY and HNC approximations. For hard spheres, the MSA is identical to the PY approximation. However, it is more accurate for many other systems, including charged and dipolar hard spheres. The linearization inherent in the MSA leads to poor results for correlation functions at low densities but the results become more accurate as the density is increased. The thermodynamic functions are usually reliable even at low densities. Often the MSA yields analytic results. The HNC approximation is generally more accurate but the equations must be solved numerically.

To go beyond these simple approximations, one must introduce the concept of the bridge function, $B_{i j}(r)$, that is defined by

$$
\gamma_{i j}(r)=\ln y_{i j}(r)-B_{i j}(r)
$$

Quite simply, the bridge function is everything missing from the $\mathrm{HNC}$ approximation. In terms of the bridge function, the HNC approximation is

$$
B_{i j}(r)=0
$$

and the PY approximation is

$$
B_{i j}(r)=y_{i j}(r)-1-\ln y_{i j}(r)
$$

Other bridge functions have been suggested. For example, the Martynov - Sarkisov (MS) approximation [6] is

$$
B_{i j}(r)=-\frac{1}{2}\left[\ln y_{i j}(r)\right]^{2}
$$


and the Verlet closure [7] is

$$
B_{i j}(r)=-\frac{1}{2} \cdot \frac{\gamma_{i j}^{2}(r)}{1+\alpha_{i j} \gamma_{i j}(r)}
$$

Verlet used the constant value $\alpha_{i j}=0.8$. Henderson et al. [8] suggested that $\alpha_{i j}$ should be state dependent. They proposed for a single component hard sphere fluid

$$
\alpha=17 / 120 \eta+0.5150-0.2210 \eta,
$$

where

$$
\eta=\frac{\pi \rho d^{3}}{6}
$$

and $d$ is the hard sphere diameter.

Both the MS and modified Verlet (MV) closures are better than the HNC and PY closures (at least for hard spheres) [9]. The MV closure is the better of the two. In addition to being less successful than the MV closure, the MS closure leads to a quadratic equation for $y_{i j}(r)$ that may not have real solutions. For hard sphere mixtures, the MV closure has not been tested sufficiently. However, the preliminary results are promising [10]. A closure that is similar to the MV closure has been proposed by Duh et al. [11-12] for fluids, such as the Lennard-Jones fluid, with attractive interactions. Only the HNC and MSA closures and some variations of these closures have been tested for fluids with long range Coulomb and dipolar forces.

\section{OZ EQUATION FOR CONFINED FLUIDS AND COLLOIDS}

Following Henderson, et al. (HAB) [13] consider the $\mathrm{OZ}$ equation for the particular situation where one particle is exceedingly large but present in small concentration. In this situation, THE OZ becomes a set of three equations

$$
h_{b b}\left(r_{12}\right)-c_{b b}\left(r_{12}\right)=\rho_{b} \int d \mathbf{r}_{3} h_{b b}\left(r_{13}\right) c_{b b}\left(r_{32}\right),
$$

and

$$
h_{b c}\left(r_{12}\right)-c_{b c}\left(r_{12}\right)=\rho_{b} \int d \mathbf{r}_{3} h_{b c}\left(r_{13}\right) c_{b b}\left(r_{32}\right),
$$

and

$$
h_{c c}\left(r_{12}\right)-c_{c c}\left(r_{12}\right)=\rho_{b} \int d \mathbf{r}_{3} h_{b c}\left(r_{13}\right) c_{b c}\left(r_{32}\right),
$$

The subscript $b$ denotes the bulk (supporting) fluid that consists of small particles. For a simpler notation, the subscript $b$ will be suppressed. The subscript $c$ denotes the large colloidal particles, whose density has been assumed to be vanishingly small. In the extreme case, where the size of the large particle is infinite, the species $c$ is a surface or wall.

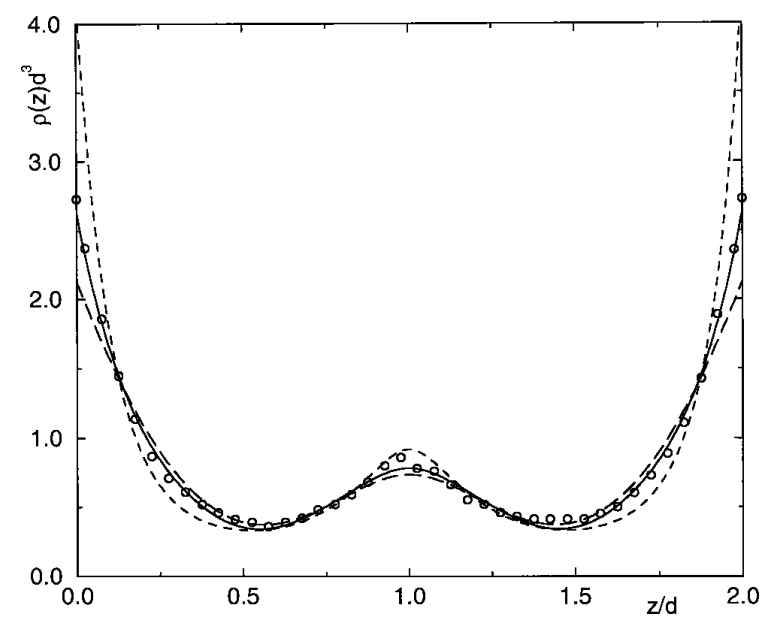

Fig. 1. Density profiles of a hard sphere fluid in a pore of width $L=2 d$. The fluid is in equilibrium with a hard sphere fluid whose density is $\rho d^{3}=0.6$. The parameter $L$ is the separation of the two distances of closest approach. The solid, long dashed and short dashed curves give the MV, PY, and HNC, results, respectively. The circles give the simulation results[17].

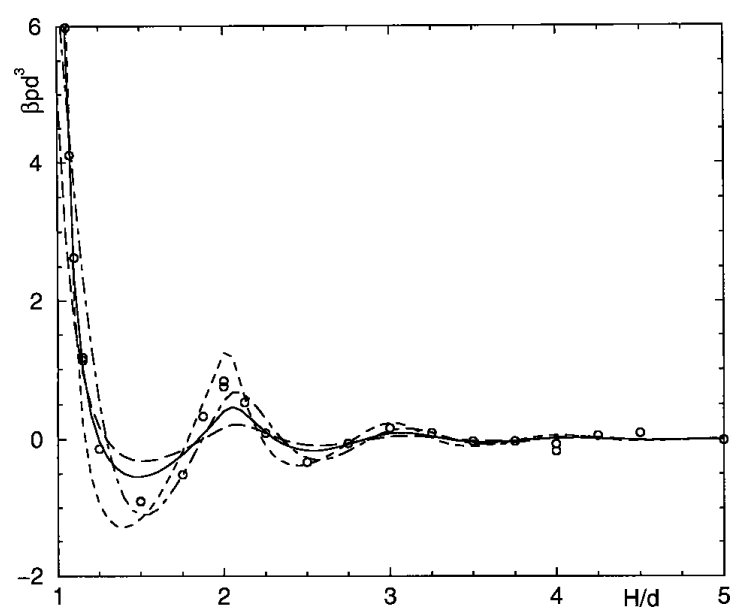

Fig. 2. Pressure on two walls separated by a distance $H=L+d$ with a hard sphere fluid between the walls. The fluid is in equilibrium with a hard sphere fluid whose density is $\rho d^{3}=0.6$. The solid, long dashed and short dashed curves and circles have the same meaning as in Fig. 1. The long-short dashed curve gives the results of eqs. (31) and (32).

Note that eqs. (18)-(20) are partially decoupled. Equation (18) can be solved without reference to eqs. (19) and (20) and gives the correlation function of the bulk fluid. 
Once eq. (18) has been solved, eq. (19) can be solved without reference to eq. (20). Once eqs. (18) and (19) have been solved, eq. (20) may be solved.

If the size of the large particles is very large, HAB showed that eq. (19) becomes

$$
h_{c}\left(z_{1}\right)-c_{c}\left(z_{1}\right)=\rho \int d z_{2} h_{c}\left(z_{2}\right) C\left(\left|z_{1}-z_{2}\right|\right)
$$

where $z_{i}$ is the distance of a small particle from the surface (not the center) of the large particle and

$$
C(s)=2 \pi \int d t t c(t)
$$

The density profile near the large particle (or surface) is given by

$$
\rho(z)=\rho\left[1+h_{c}(z)\right]
$$

Note that $\rho(\infty)=\rho$, as expected. Other profiles, such as charge profiles, can be constructed from $h_{c}(z)$.

HAB solved eq. (21) for hard spheres near a hard wall using the PY closure. The PY profile is in reasonable agreement with simulation data [14] but is too small at contact with the surface or colloidal particle. In fact, in the PY approximation, the contact value of the profile of a fluid near a hard wall is given by

$$
g_{c}(d / 2)=\left(\frac{\beta \partial p}{\partial \rho}\right)^{1 / 2}
$$

which is small compared to the exact result, obtained from force balance considerations,

$$
g_{c}(d / 2)=\frac{\beta p}{\rho}
$$

On the other hand, the HNC results are too large. More recently [15], we have calculated the density profiles for hard spheres near a hard wall using the MV closure. The agreement with the simulation results is good.

Lozada Cassou [16] has shown that eq. (21), can be applied to a fluid in a pore formed by two surfaces. The density profile of a hard sphere fluid in a pore formed by two hard walls is shown in fig. 1 . The MV closure results [15] agree well with those obtained by simulations [17].

The pressure, $p$, or solvation force per unit area, acting on the walls of the pore is given by

$$
\beta p=\rho(d / 2 ; L)-\rho(d / 2, \infty)
$$

where $L$ is the width of the pore. The quantities $\rho(d / 2 ; L)$ and $\rho(d / 2, \infty)$ are the contact values of the profile for separations $L$ and $\infty$, respectively. The PY, HNC and MV results for $p$ are compared with simulation [17] in fig. 2. None of these theories is really satisfactory.

\section{THE INTERACTION BETWEEN COLLOIDAL PARTICLES}

The interaction between colloidal particles can be obtained from eq. (20) since

$$
W_{c c}(x)=-k T \ln g_{c c}(x)
$$

If the colloidal particle is large, eq. (20) becomes

$$
h_{c c}(x)-c_{c c}(x)=\pi \rho a \int h_{c}(y) d y \int c_{c}(t) d t
$$

and the solvation force between the colloidal particles can be obtained from

$$
F_{c c}(x)=-\frac{\partial \beta W_{c c}(x)}{\partial x}
$$

Thus,

$$
\begin{aligned}
F_{c c}(x) & =-\frac{\partial \beta V_{c c}(x)}{\partial x}+\frac{\partial B_{c c}(x)}{\partial x} \\
& -\pi \rho a \int h_{c c}(y) c_{c c}(x-y) d y
\end{aligned}
$$

where $V_{c c}(x)$ is the interaction between the large particles.

Notice that the last term in eq. (30) is proportional to $a$. In the known cases, $V_{c c}(x)$ is proportional to $a$. We presume that this is a general feature. If we presume that a good approximation for $B_{c c}(x)$ would give $B_{c c}(x)$ proportional to $a$, we may conclude that $F_{c c}(x)$ is proportional to $a$, in accord with eq. (1). Further notice that, in contrast to eqs. (18) or (19), no iteration is required in any numerical solution of eqs. (20) or (28) or (29).

Obviously, when $a$ is large, there is a close similarity between the geometry of the slit and the region between the colloidal particles. Thus, the solvation force between two colloidal particles can be obtained also by calculating the pressure acting on the walls of a pore, using eqs. (19) or (21) together with the Derjaguin approximation

$$
\frac{F_{c c}(x)}{a}=\frac{\pi E(L)}{A}
$$

where $E(L) / A$ is the solvation energy/unit area between the two walls of the slit. Again notice that $F_{c c}(x)$ is proportional to $a$. Thus, there are two methods of calculating $F_{c c}(x)$ or $E(L)$ and $p$, one using eqs. (19) or (21) with eq. (31) and using eqs. (20) or (28) or (30).

The electrostatic portion of the DLVO theory can be obtained by applying the solution of the MSA for point charged hard spheres to either of these routes. A little 
care must be used if the latter route is used. Equations (18) and (19) are solved in the MSA but eq. (20) must be solved in the HNC approximation. We can call this hybrid approximation the HNC/MSA/MSA approximation. If the MSA were used throughout, $g_{c c}(x)$ would be proportional to $a$ and $F_{c c}(x)$ would be proportional to $\ln a$, an unphysical result. Such a hybrid approximation has been used by Mitchell, et al. [18] and by Henderson and Lozada-Cassou (HLC) [19].

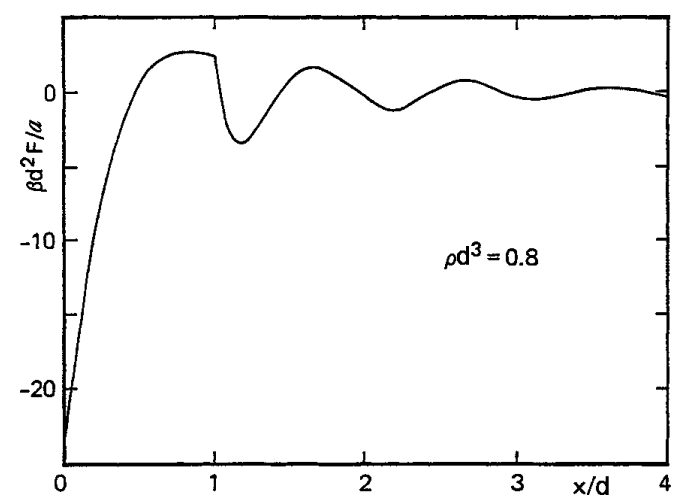

Fig. 3. The force between two exceedingly large spheres of diameter $2 a$ in a fluid of small spheres of diameter $d$ whose density is $\rho d^{3}=0.8$, calculated using the HNC/PY/PY approximation.

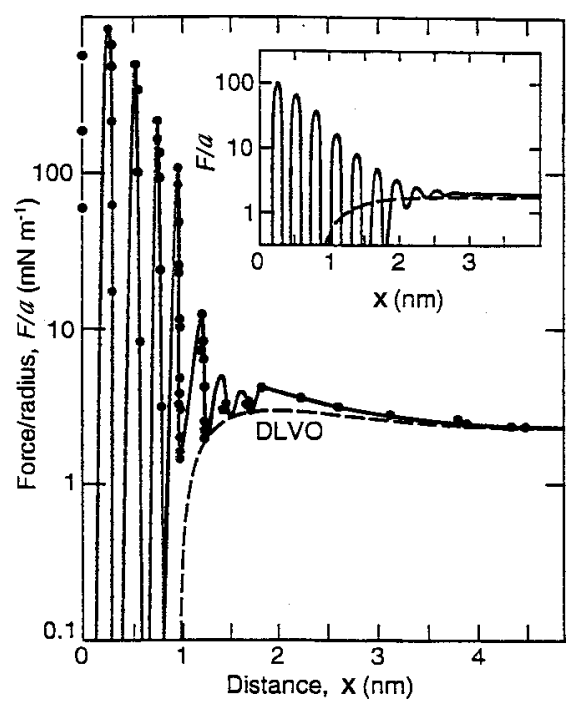

Fig. 4. A comparison of experiment and HLC theory (insert) of the force between two curves macroscopic surfaces, immersed in a aqueous $10^{-3} \mathrm{M} \mathrm{KCl}$ solution. Reproduced with permission from reference 1 .

These two results give a means of generalizing (and making systematic) the DLVO theory. One term that is missing is the effect of the excluded volume of the supporting fluid. This is called the depletion force in colloidal science and the excluded volume force in statistical me- chanics. An approximate expression for this force can be obtained by assuming that the supporting fluid is hard sphere fluid (with a dielectric constant to be sure). The Laplace transform of this volume exclusion or depletion force can be obtained analytically. However, the inversion of the Laplace transform is approximate. The force between two large spheres in a supporting fluid of small spheres is given in fig. 3 .

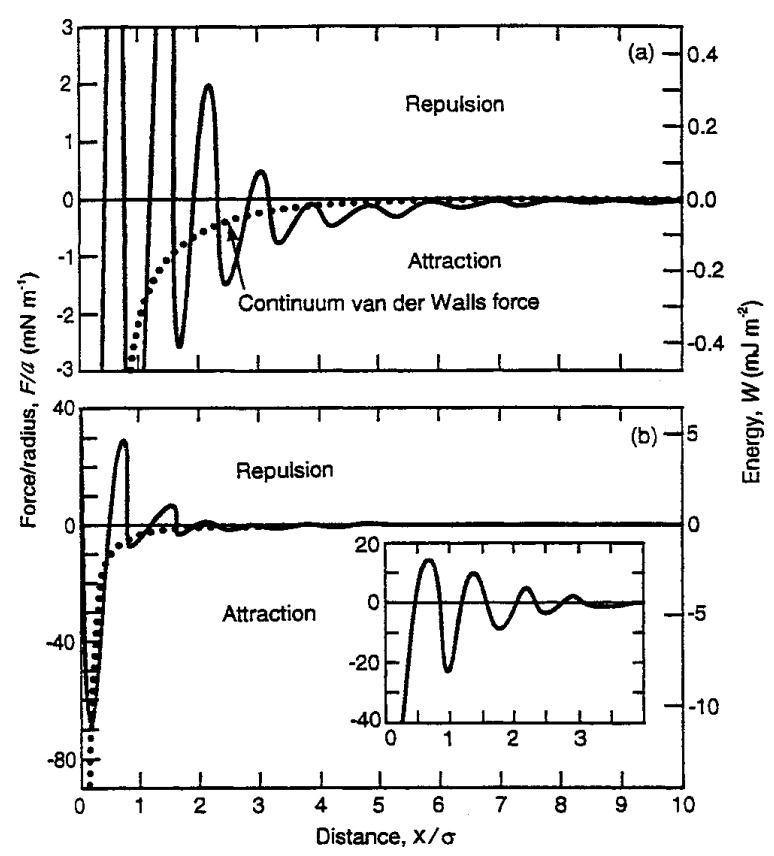

Fig. 5. Comparison of experiment and the HLC theory (insert) of the force between two curved macroscopic surfaces immersed in octamethylcyclotetrasiloxane. Reproduced with permission from reference 1 .

Although no simple results for $W_{c c}(x)$ and $F_{c c}(x)$ exist, the contact value of $W_{c c}(x)$ can be obtained. Thus, for the solvation interaction at contact between two large spheres in a fluid of small spheres,

$$
-\beta W_{c c}(x)=\ln g_{c c}\left(d_{c c}\right)=\frac{3 \eta R}{2(1-\eta)^{2}}
$$

in the HNC/PY/PY scheme. In eq. (32), $R=d_{c c} / d$.

This procedure can be checked by using the Derjaguin approximation to obtain the solvation pressure on the hard walls of a pore containing a hard sphere fluid. The agreement with the simulation results, shown in fig. 2, is surprisingly good. The depletion or volume exclusion force, obtained by HLC, can be added to the DLVO terms, already given in eq. (1); this is in the spirit of the DLVO theory where terms calculated separately are added together, assuming a kind of superposition approximation. It is unclear whether this is valid. Indeed, its validity has not been tested. For the moment, we accept this is a working hypothesis. 
In addition to the depletion force, HLC estimated the effect of dielectric saturation of the solvent dipoles in the vicinity of the colloidal particles and added this term to obtain a four term expansion for $F_{c c}(x)$. Some of their results are plotted in figs. 4 and 5 . The agreement with experiment is very good, especially when the experimental results are corrected (roughly) for the effect of the elastic deformation of the large particles.

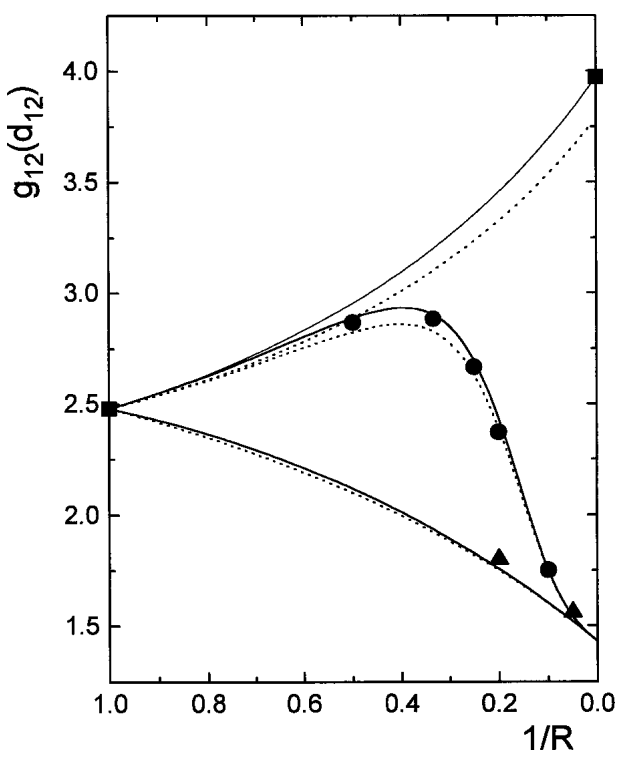

Fig. 6. $g_{12}\left(d_{12}\right)$ as a function of $1 / R=d_{11} / d_{22}$ for a hard sphere mixture for $\delta_{3}=0.3$. The solid and dashed curves give the results of eq. (34) and (39), respectively. The points give simulation results. The three sets of curves are for $x_{2}=1,0.001$ and 0 .

\section{HARD SPHERE MIXTURES}

The HNC/PY/PY scheme is useful. However, away from the special limit $\rho_{c} \rightarrow 0$ and $a \rightarrow \infty$, the use of the PY closure for eq. (20) seems accurate. Lebowitz [20] has obtained an analytical expression for the Laplace transform of the PY $g_{i j}(r)$. Again the inversion is approximate. However, the analytic expression for the contact values are known; they are

$$
g_{i j}\left(d_{i j}\right)=\frac{1}{1-\delta_{3}}+\frac{d_{i i} d_{j j}}{d_{i j}} \frac{3 \delta_{2}}{2\left(1-\delta_{3}\right)^{2}},
$$

where $d_{i j}=\left(d_{i i}+d_{j j}\right) / 2$. Away from $\rho_{c} \approx 0$, these expressions are fairly good. An extension due to Boublik and Mansoori et al. [21] (BMCLS),

$$
g_{i j}\left(d_{i j}\right)=\frac{1}{1-\delta_{3}}+\left(\frac{d_{i i} d_{j j}}{d_{i j}}\right) \frac{3 \delta_{2}}{2\left(1-\delta_{3}\right)^{2}}
$$

$$
+\left(\frac{d_{i i} d_{j j}}{d_{i j}}\right)^{2} \frac{\delta_{2}^{2}}{2\left(1-\delta_{3}\right)^{3}}
$$

is even more accurate. In the above equations

$$
\delta_{n}=\pi \rho\left(x_{1} d_{11}^{n}+x_{2} d_{22}^{n}\right) / 6,
$$

where

$$
\rho=\sum_{i} \rho_{i}
$$

and

$$
x_{i}=\rho_{i} / \rho .
$$

The index 1, or $b$, denotes the bulk (small) hard sphere fluid and the index 2, or $c$, denotes the large hard spheres.

It is evident that neither eq. (33) or (34) are applicable in the limit $\rho_{c} \rightarrow 0$ since they would give $W_{c c}(x)$ proportional to $\ln d_{22}$ and not $d_{22}$. Hopefully, by now we are convinced that this is incorrect. Henderson et al. [10, $22]$ have constructed the formulae

$$
g_{11}\left(d_{11}\right)=g_{11}^{B M C S L}\left(d_{11}\right),
$$

and

$$
\begin{aligned}
g_{12}\left(d_{12}\right) & =g_{12}^{B M C S L}\left(d_{12}\right)+d_{11}^{2} \frac{\delta_{2}^{2}\left(R^{2}-1\right)}{\left(1-\delta_{3}\right)^{2}(R+1)^{3}} \\
& -d_{11}^{3} \frac{\delta_{2}^{3}\left(R^{3}-1\right)}{\left(1-\delta_{3}\right)^{3}(R+1)^{3}},
\end{aligned}
$$

and

$$
\begin{aligned}
g_{22}\left(d_{22}\right) & =\frac{1}{1-\delta_{3}}+\frac{3 \delta_{3}}{2\left(1-\delta_{3}\right)^{2}}+\frac{\delta_{3}^{2}}{2\left(1-\delta_{3}\right)^{3}} \\
& +\exp \left[\frac{3\left(\delta_{2} R d_{11}-\delta_{3}\right)}{2\left(1-\delta_{3}\right)^{2}}\right]-1,
\end{aligned}
$$

where $R=d_{22} / d_{11}$, as before. These expressions were not derived in any systematic manner. They are only plausible extensions that yield eq. (25) and

$$
\ln g_{22}\left(d_{22}\right) \sim d_{22}
$$

Away from $\rho_{c}=0$, eqs. (39)-(40) are about the same accuracy as the BMCSL expressions. It is only for small $\rho_{c}$ and large $d_{c c}$ that differences appear. Yau et al. [23] have tested eqs. (39) $-(40)$ by performing simulations for $\rho_{c}$ as small as 0.01. As is seen in figs. 6 and 7 , eqs. (39)-(40) are surprisingly accurate.

There is no way that $\rho_{c}=0$ can be reached by simulation. However, it can be reached by means of integral 
equations. We have already reported PY/PY/PY and HNC/PY/PY results. Regrettably, a reliable expression for $B_{c c}$ has not been devised. More sophisticated theories, based on higher order OZ equations are a possible approach. Henderson et al.[24] have made such a calculation. This second order theory is rather difficult but is feasible for $\rho_{c}=0$. A comparison with eqs. (39) and (40) is given in figs. 8 and 9 . The agreement is good.

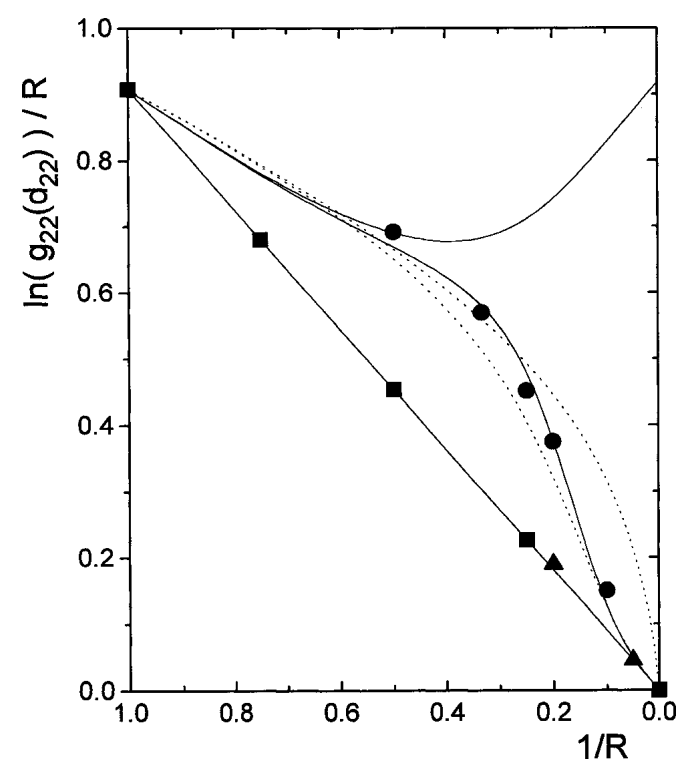

Fig. 7. $g_{22}\left(d_{22}\right)$ as a function of $1 / R=d_{11} / d_{22}$ for a hard sphere mixture for $\delta_{3}=0.3$. The solid and dashed curves give the results of eq. (34) and (39), respectively. The points give simulation results. The three sets of curves are for $x_{2}=1,0.001$ and 0 .

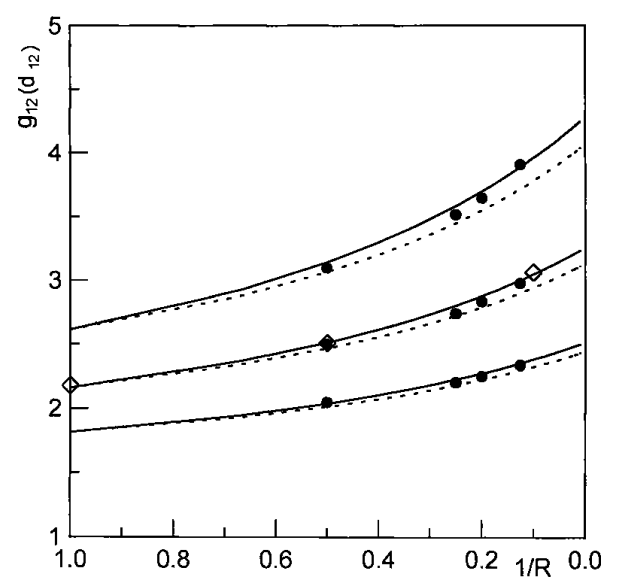

Fig. 8. $g_{12}\left(d_{12}\right)$ as a function of $1 / R=d_{11} / d_{22}$ for an exceedingly small number of large hard spheres $\left(x_{2}=0\right)$ in a solvent of small hard spheres. The three sets of curves are for $\rho d_{11}=0.4,0.5$, and 0.6 , respectively. The solid and broken curves give the results of eqs. (39) and (40). The circles and diamonds gives the results of a highly accurate second order integral equation.
Henderson et al. [25] have used eqs. (38)-(40) to examine the question of phase separation in hard sphere mixtures. They find that phase separation occurs at small $\rho_{c}$. Some years ago, Lebowitz and Rowlinson [26] used the PY theory to examine this question and concluded that it did not occur. However, their calculation is only as good as the PY theory, which fails at small $\rho_{c}$. Ise et al. [27] have observed 'voids' in colloidal suspensions when the concentration of the colloidal particles is small. It seems probable that these 'voids' are due to phase separation (not to be confused with flocculation). Independently, Wasan et al.[28] have obtained experimental and theoretical evidence for phase separation in colloidal suspensions.

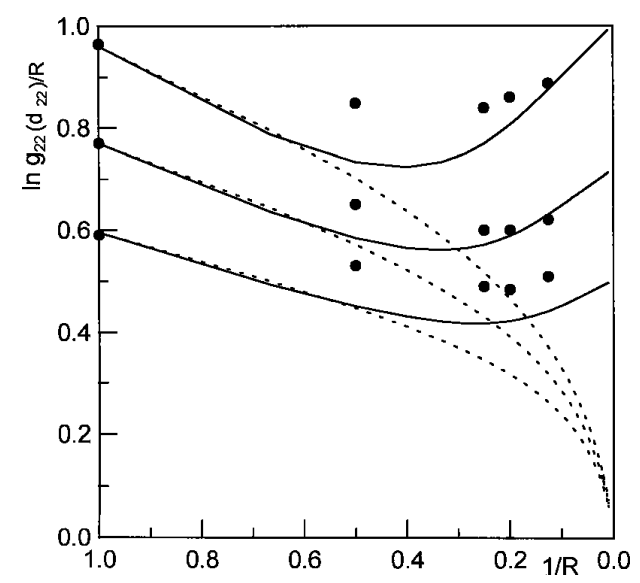

Fig. 9. $g_{22}\left(d_{22}\right)$ as a function of $1 / R=d_{11} / d_{22}$ for an exceedingly small number of large hard spheres $\left(x_{2}=0\right)$ in a solvent of small hard spheres. The curves and circles have the same meaning as in Figure 8.

\section{SUMMARY}

The neglect of depletion or volume exclusion forces is a major deficiency in the DLVO theory. fairly good expression for these forces are available, at least for hard spheres. What is lacking is a reliable approximation for the bridge function, $B_{c c}(x)$, between the colloidal particles. This is one thrust of our current research. Possibly,

$$
B_{c c}(x)=0
$$

is valid for $\rho_{c}=0 a$ being large. Certainly, the results presented here indicate this. Even so, some reasonable way of passing from eq. (42) to some other closure away this region is needed. Once the question of a closure is settled, it would be desirable to solve the $\mathrm{OZ}$ equation with the hard core, van der Waals, and Coulomb interactions all combined. This way of ad hoc superposition of results can be tested and, if need be, replaced. Already, the results presented here show that the DLVO theory is 
incomplete. It is reasonable to expect that a more sophisticated theory for colloids will emerge. This is partly true already. The related problem of a fluid in pores of various shape is of interest. The exploration of the theory of such systems has only begun.

\title{
ACKNOWLEDGMENT
}

This work was supported in part by the National Science Foundation (Grant CHE96-01971) and by the donors of the Petroleum Research Fund that is administered by the American Chemical Society (Grant ACS-PRF-31573-AC9).

[1] J. N. Israelachvili, Intermolecular and Surface Forces (2nd Edition, Academic Press, London, 1992).

[2] A. D. Nikolov, D. T. Wasan, J. Colloid Interface Sci. 133, 1 (1989); X. L. Chu, A. D. Nikolov, D. T. Wassan, Langmuir 10, 4403 (1994).

[3] The HNC approximation was proposed by several authors. For a full set of references, see J. A. Barker, D. Henderson, Rev. Mod. Phys. 48, 587 (1976).

[4] J. K. Percus, G. J. Yevick, Phys. Rev. 110, 1 (1958).

[5] J. L. Lebovitz, J. K. Percus, Phys. Rev. 144, 251 (1966).

[6] G. A. Martynov, G. N. Sarkisov, Mol. Phys. 49, 1495 (1983).

[7] L. Verlet, Mol. Phys. 41, 183 (1980).

[8] D. Henderson, K. Y. Chan, L. Degreve, J. Chem. Phys., 101, 6975 (1994).

[9] M. Beard, D. Henderson, Mol. Phys. (in press)

[10] D. Henderson, A. Malijevsky, S. Labik, K. Y. Chan, Mol. Phys. 87, 273 (1996).

[11] D. M. Duh, A. D. J. Haymet, J. Chem. Phys. 97, 7716 (1992); 103, 2625 (1995).

[12] D. M. Duh, D. Henderson, J. Chem. Phys. 104, 6742 (1996).

[13] D. Henderson, F. F. Abraham, J. A. Barker, Mol. Phys. 31, 1291 (1976).

[14] I. K. Snook, D. Henderson, J. Chem. Phys. 68, 2134 (1978); J. R. Henderson, F. van Swol, Mol. Phys. 51, 991 (1984).

[15] D. Henderson, S. Sokolowski, D. Wassan, J. Phys. Chem. (submitted).
[16] M. Lozada-Cassou, in: Fundamentals of Inhomogeneous Fluids, edited by D. Henderson (Dekker, New York, 1992).

[17] M. S. Wertheim, L. Blum, D. Bratko, in: Micellar Solutions and Microemulsions, edited by S. H. Chen, R. Rajagopalan (Springer-Verlag, 1990).

[18] D. J. Mitchell, B. W. N. Ninham, B. A. Pailthorpe, J. Chem. Soc. Faraday Trans. II 74, 1098, 1116 (1978); D. Y. C. Chan, D. J. Mitchell, B. W. N. Ninham, Mol. Phys. 351669 (1978); Chem. Phys. Lett. 56, 533 (1978).

[19] D. Henderson, M. Lozada-Cassou, J. Colloid Interface Sci. 114, 180 (1986); D. Henderson, ibid 121, 486 (1988).

[20] J. L. Lebowitz, Phys. Rev. 133, A895 (1964).

[21] T. Boublik, J. Chem. Phys. 53, 471 (1970); G. A. Mansoori, N. F. Carnahan, K. E. Starling, T. W. Leland, Jr., ibid 54, 1523 (1971).

[22] D. H. L. Yau, K. Y. Chan, D. Henderson, Mol. Phys. 88, 1237 (1996).

[23] D. H. L. Yau, K. Y. Chan, D. Henderson, Mol. Phys. 91, 1137 (1997).

[24] D. Henderson, S. Sokolowski, D. Wassan, Mol. Phys. (in press).

[25] D. Henderson, D. Boda, K. Y. Chan, D. Wassan, Mol. Phys. (in press).

[26] J. L. Lebowitz, J. S. Rowlinson, J. Chem. Phys. 41, 133 (1964).

[27] N. Ise, et al, Langmuir 8, 2952 (1992); 9, 394 (1993); 11, 2853 (1995); J. Chem. Phys. 103, 10146 (1995).

[28] X. Wu, A. D. Nikolov, D. Wassan, AIChE J. (in press).

\section{СИЛИ ЗА РАХУНОК ВИКЛЮЧЕНОГО ОБ`ЄМУ В КОЛОЇДНИХ СУСПЕНЗІЯХ}

\author{
Д. Гендерсон ${ }^{1}$, С. Соколовскі ${ }^{2}$, Д. Васан ${ }^{3}$ \\ ${ }^{1}$ Відділ хемій та біологї Університету Брі гема Ян а, Прово, Юта, 84602 СІІІА \\ ${ }^{2}$ Bідділ моделювання фізико-хемічних прочесів Університету, \\ Марї̈ Кюрі Склодовськой, Люблін, 200 З1, Польща \\ ${ }^{3}$ Технологічний інститут Іллінойсу, Чика о, 60616, СIIIA
}

\begin{abstract}
Класичну теорію взаємодії між колоїдами розвинули Дерягін, Ландау, Вервей і Овербек. У ній допускається, що взаємодія складається $з$ далекося жного електростатичного доданка і притягального доданка дисперсійного типу. Бракує ва жллвого доданка, що описує сили виключеного об' єму. Набли жено цей доданок одержали Гендерсон і Лозада Кассоу з теорії сумішей твердих кульок. Попередньо дискутовано деякі неузгодженості у способі отримання доданка, ві дпові да.льного за сили виключеного об' єму. Обговорено певні аспекти цього питання. Також оглянуто недавні роботи, у яких розв'язано цю проблему і які підтверджують правильність оригінального результату теорії Гендерсона і Лозада Кассоу.
\end{abstract}

